\title{
A Method of Computing Exact Inverses of Matrices With Integer Coefficients ${ }^{1}$
}

\author{
J. Barkley Rosser ${ }^{2}$
}

\begin{abstract}
In theory, the problem of computing the exact inverse of a matrix $A$ with integer coefficients is completely solved by solving exactly the simultaneous equations $A x=y$, in which both $x$ and $y$ are variable vectors. This solution can be carried out by any one of numerous well-known procedures, resulting in expressions for the components of $x$ as linear combinations of the components of $y$. The coefficients of these linear combinations are just the components of $A^{-1}$ because we have $x=A^{-1} y$.

In actual practice, if the order of $A$ is at all large, the exact components of $A^{-1}$ will be fractions whose numerator and denominator each have a large number of digits, and the usual methods of solution become extremely laborious due to the necessity for carrying an even larger number of significant digits throughout most of the computation. In the method presented herein, the number of significant digits involved builds up gradually, and only the final stages of the computation involve a large number of digits. Moreover, the method can be readily adapted to use on IBM equipment, and so all but the final stages (in which many significant digits must be carried) can be readily mechanized.
\end{abstract}

\section{Illustration of a Solution by Previous Methods}

Suppose we require the exact inverse of

$$
A=\left|\begin{array}{rrrrrr}
152 & -128 & 183 & 83 & -141 & -27 \\
103 & -89 & 156 & -91 & 135 & -96 \\
72 & 195 & 75 & 113 & -187 & 178 \\
157 & -192 & -37 & -138 & 71 & -179 \\
34 & 190 & -120 & 102 & 37 & 65 \\
191 & 77 & -154 & 117 & -131 & -112
\end{array}\right|
$$

To invert this, we write

$$
\begin{aligned}
152 x_{1}-128 x_{2}+183 x_{3}+83 x_{4}-141 x_{5}-27 x_{6} & =y_{1} \\
103 x_{1}-89 x_{2}+156 x_{3}-91 x_{4}+135 x_{5}-96 x_{6} & =y_{2} \\
72 x_{1}+195 x_{2}+75 x_{3}+113 x_{4}-187 x_{5}+178 x_{6} & =y_{3} \\
157 x_{1}-192 x_{2}-37 x_{3}-138 x_{4}+71 x_{5}-179 x_{6} & =y_{4} \\
34 x_{1}+190 x_{2}-120 x_{3}+102 x_{4}+37 x_{5}+65 x_{6} & =y_{5} \\
191 x_{1}+77 x_{2}-154 x_{3}+117 x_{4}-131 x_{5}-112 x_{6} & =y_{6} .
\end{aligned}
$$

Eliminating $x_{1}$ from the last five of these by use of eq 1 gives

$$
\begin{array}{r}
344 x_{2}-4863 x_{3}+22381 x_{4}-35043 x_{5}+11811 x_{6}=103 y_{1}-152 y_{2} \\
-4857 x_{2}+222 x_{3}-1400 x_{4}+2284 x_{5}-3625 x_{6}=9 y_{1}-19 y_{3} \\
9088 x_{2}+34355 x_{3}+34007 x_{4}-32929 x_{5}+22969 x_{6}=157 y_{1}-152 y_{4} \\
-16616 x_{2}+12231 x_{3}-6341 x_{4}-5209 x_{5}-5399 x_{6}=17 y_{1}-76 y_{5} \\
-36152 x_{2}+58361 x_{3}-1931 x_{4}-7019 x_{5}+11867 x_{6}=191 y_{1}-152 y_{6} .
\end{array}
$$

\footnotetext{
1 The preparation of this paper was sponsored (in part) by the Office of Naval Research.
}

${ }^{2}$ National Bureau of Standards and University of California at Los Angeles. 
The multipliers that were used in eliminating $x_{1}$ are apparent from the coefficients of the $y$ 's on the right sides of the reduced equations.

We now use eq 2 to eliminate $x_{2}$ from the four equations that follow it. The multipliers we use for eq 2 are, respectively, 4857, 1136, 2077, and 4519. We get

$$
\begin{aligned}
& -23543223 x_{3}+108222917 x_{4}-169418155 x_{5}+56119027 x_{6}=503367 y_{1}-738264 y_{2}-6536 y_{3} \\
& -7001633 x_{3}+23962515 x_{4}-38392901 x_{5}+12429629 x_{6}=110257 y_{1}-172672 y_{2}+6536 y_{4} \\
& -9574518 x_{3}+46212674 x_{4}-73008298 x_{5}+24299290 x_{6}=214662 y_{1}-315704 y_{2}-3268 y_{5} \\
& -19466374 x_{3}+101056706 x_{4}-158661134 x_{5}+53884190 x_{6}=473670 y_{1}-686888 y_{2}-6536 y_{6} .
\end{aligned}
$$

We now use eq 3 to eliminate $x_{3}$ from the three equations that follow it. The multipliers we use for eq 3 are, respectively, 3 68507, 167974 , and 1024546 . We get

$$
\begin{array}{rl}
10188542775664 x_{4}-14858479736168 x_{5}+52784896 & 85096 x_{6}=48872940000 y_{1} \\
& -58094641224 y_{2}-2408561752 y_{3}-8098868712 y_{4} \\
-909000396128 x_{4}+1697429229652 x_{5}-6100170 & 01012 x_{6}=-4111209360 y_{1} \\
+ & 6388907320 y_{2}-1097878064 y_{3}+1349811452 y_{5} \\
-14341725647920 x_{4}+23023015346048 x_{5}-92722912 & 23488 x_{6}=-71209903008 y_{1} \\
+94749169752 y_{2}-6696432656 y_{3}+8098868712 y_{6} .
\end{array}
$$

We now use eq 4 to eliminate $x_{4}$ from the two equations that follow jt. The multipliers we use for eq 4 are, respectively, 6321634 and 99739385 . We get

$26343373406971322772 x_{5}-9854756133743223540 x_{6}=17652507760304880 y_{1}+85440105388636424 y_{2}$ -9301742 $2417060256 y_{3}-51198083811315408 y_{4}+95642398170851884 y_{5}$,

$149345838132013998936 x_{5}-130525236869220170136 x_{6}=-171100240351599936 y_{1}$

+919234470123165744y $y_{2}-714711683635159272 y_{3}-807776184530622120 y_{4}+573854389025111304 y_{6}$.

We now use eq 5 to eliminate $x_{5}$ from the equation that follows it. The multiplier we use for eq 5 is 135229110622 . We get

$1780807616783302242949552975104 x_{6}=6468438145668501099175688544 y_{1}$

-103727853 $5914981148 \quad 61699 \quad 71008 y_{2}+446 \quad 95683 \quad 03519 \quad 19158 \quad 33166 \quad 05736 y_{3}$

$+12344655651847480586283954504 y_{4}+12933636442399499877413111848 y_{5}$

$-13688320420859690187835706376 y_{6}$.

Dividing this through by the common factor 191284796341703768 , we get $9309718549728 x_{6}=33815746308 y_{1}-54226920056 y_{2}+23366040527 y_{3}+64535477403 y_{4}+67614555311 y_{5}$ $-71559897507 y_{6}$.

Substituting this back into eq $5,4,3,2$, and 1 , we get $9309718549728 x_{5}=18888462180 y_{1}+9908763656 y_{2}-24131282729 y_{3}$ $+6048642243 y_{4}+59093751511 y_{5}-26769743115 y_{6}$, $9309718549728 x_{4}=\quad 54684079248 y_{1}-10539226192 y_{2}-49498208180 y_{3}$ $-32013843300 y_{4}+51149667628 y_{5}-1965885132 y_{6}$, $9309718549728 x_{3}=-2993479728 y_{1}+42923511632 y_{2}+\quad 4398917764 y_{3}$ $-36856173708 y_{4}-28947823484 y_{5}+13025185404 y_{6}$ $9309718549728 x_{2}=-49505983764 y_{1}+50131385144 y_{2}+22100258797 y_{3}$ $-37778195727 y_{4}-38741730707 y_{5}+41981957079 y_{6}$ $9309718549728 x_{1}=16830783936 y_{1}-4147504752 y_{2}+22108912392 y_{3}$ $+47115369576 y_{4}+41124340200 y_{5}-16798690584 y_{6}$ From these results one can easily write down $A^{-1}$. 
We hardly need to stress the fact that the procedure outlined above is not a really practicable method to find $A^{-1}$. In the present case, we did carry the computation through to the bitter end, just to show how unwieldy it becomes, but it required 38 hours of computing time by a trained professional computer. We might note further that the computation would have been even more unwieldy and extensive if we had not deviated from a strict mechanical procedure by removing common factors from our multipliers when combining equations. Precisely, what we did do in this direction is described below.

In general, if we wish to eliminate $x_{i}$ from two equations $a x_{i}+\ldots, b x_{i}+\ldots$, the mechanical way to proceed is to multiply the first equation by $b$ and the second equation by $-a$, and add. However, in order to mitigate somewhat the frightful increase in the sizes of the coefficients in the later stages of the process, we have in every such step of the present computation determined the greatest common factor, $c$, of $a$ and $b$, and have multiplied the first equation by $b / c$ and the second by $-(a / c)$, and added. For example, in the case of eliminating $x_{5}$ from eq 5 and the equation following it, $a$ is a 20-digit number and $b$ is a 21-digit number. However, the common factor, $c$, is a 10 -digit number, so that our multipliers, $b / c$ and $-(a / c)$, are 12- and 11-digit numbers, respectively. If we had used $b$ and $-a$ as multipliers, the coefficients in eq 6 would each have had about nine more digits.

Similarly, in the multipliers used with eq 4, a six-digit factor was removed, and in the multipliers used with eq 3 a two-digit factor was removed. Without such removal of factors, the coefficients of eq 6 would each have had about 15 more digits, so that it is doubtless worth while to carry out such a determination of common factors. Nevertheless, this portion of the computation can be quite a chore, especially when (as in the present case) the greatest common factor of a 20-digit number and a 21-digit number is required. Also, if determination of common factors is carried out, mechanization of the process is more much difficult.

One could effect a further saving in the number of digits carried by extracting common factors from each of the equations derived in the course of the computation, instead of only from eq 6. However, this entails a great increase in labor with only a moderate decrease in the number of digits in the various equations. In the present case, if all possible common factors would be extracted from previous equations, one would still obtain as the equation corresponding to eq 6 an equation with a 23-digit coefficient for $x_{6}$, and there would be much additional labor in the determination and extraction of common factors.

\section{A New Method of Solution}

We treat the same matrix $A$ as in the previous section. If $G$ is the inverse of $A$, then $G A$ is the unit matrix. To find $G$, we find in succession $B, C, D, E$, and $F$ with the properties that $B A$ has its first column the same as the unit matrix, $C A$ has its first two columns the same as the unit matrix, $D A$ has its first three columns the same as the unit matrix, and so on up to $G A$, which has all its columns the same as the unit matrix.

Our method for finding $B, \mathrm{C}, \ldots, G$ is a modification of the algorithm set forth in a previous note. ${ }^{3}$ In addition, we use the following well-known property of matrix multiplication. If $W$ and $V$ are matrices with $W A=V$, then if we form $W^{*}$ and $V^{*}$ from $W$ and $V$ by performing the same elementary transformation on the rows of each, we will have $W^{*} A=V^{*}$. By an elementary transformation on the rows, we mean one of (a) Multiplying the $i$ th row by a constant $\alpha$. (b) Interchanging the $i$ th and $j$ th rows. (c) Adding $\alpha$ times the $i$ th row to the $j$ th row.

By a sequence of such transformations, we can reduce $V$ to the unit matrix, and so the same sequence of transformations performed on $W$ will reduce it to $A^{-1}$. This fact is the basis of various methods for computing $A^{-1}$. The novelty in our method lies in the fact that we are able to use mainly transformation (c) with an occasional transformation (b) until the final stage of the reduction, and also that we have a mechanical procedure for keeping the sizes of the numbers small until the final stages of the reduction.

3 J. Barkley Rosser, A note on the linear Diophantine equation, Am. Math. Mo., 48662 (1941). 
As indicated earlier, we carry out the reductions on $V$ in a certain order. First we reduce the first column of $V$ to be the first column of the unit matrix. Then $W$ has been reduced to the $B$ mentioned earlier. Then, restricting ourselves to transformations that leave the first column of $V$ unchanged, we reduce the second column to be the second column of the unit matrix. Then $W$ has been reduced to the $C$ mentioned earlier. Proceeding in this way, column by column, we eventually reduce $V$ to $I$ and $W$ to $A^{-1}$.

To get started, let us take $W$ to be the unit matrix, $I$. Then $V$ is $A$. We first seek transformations that will bring the first column of $V$ to the desired form. So we temporarily ignore all other columns of $V$, and consider only the first column, which is (I). This is to be reduced to form (II).

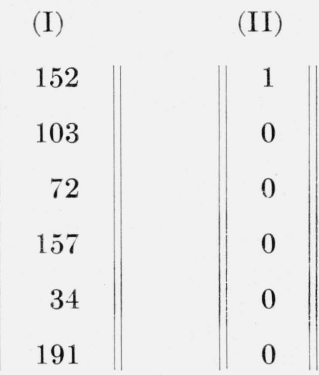

One can do this in many ways, but we follow the way that is proposed in the note referred to in footnote 3 , since this is quite mechanical but keeps the sizes of the numbers involved reasonably small. Specifically, we apply the elementary transformation (c) to those two rows containing the two numbers of maximum absolute value. Thus we first add -1 times the fourth row to the sixth, getting (III). Then we add -1 times the first row to the fourth, getting (IV). Then we add -1 times the second row to the first, getting $(\mathrm{V})$.

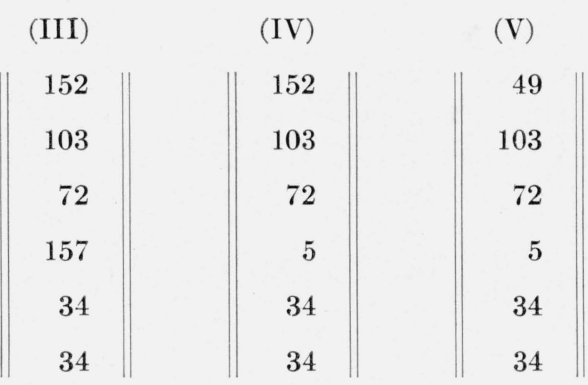

Clearly, if we were trying to reduce the sizes of the numbers as rapidly as possible, we would now add -2 times the first row to the second. However, this presupposes that good judgment is to be applied at the various steps. One of the advantages of the procedure we are describing is that it gives quite good results even when applied quite mechanically. To illustrate, we ignore the smart transformation, and proceed according to rule, adding -1 times the $i$ th row to the $j$ th for the following values of $i$ and $j$ :

$$
\begin{array}{l|l|l|l|l|l|l|l|l|l|l|l|l|l|l}
i & 3 & 1 & 5 & 5 & 2 & 3 & 1 & 2 & 2 & 1 & 4 & 5 & 1 & 1 \\
\hline j & -2 & 3 & 1 & 6 & 5 & 2 & 3 & 1 & 3 & 2 & 1 & 4 & 5 & 4
\end{array}
$$

We then have

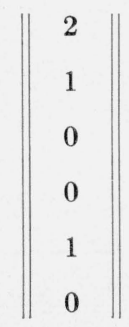


We now have a situation that occasionally arises, in which the largest number is at least twice as great as the next largest. In such case, our multiplier can conveniently be different from the -1 used uniformly so far. Thus we could conclude by adding -2 times the second row to the first, then -1 times the second row to the fifth, and, finally, by interchanging the first and second rows. However, we could equally well continue mechanically, since exactly the same result would ensue if we twice add -1 times the second row to the first row, and then add -1 times the second row to the fifth, and, finally, interchange the first and second rows.

Applying the transformations listed to the unit matrix transforms it into $B$, which is

$$
\begin{array}{||rrrrrr}
1 & 1 & -4 & 0 & 1 & 0 \\
-1 & -3 & 10 & -1 & -3 & 0 \\
-3 & 2 & 3 & 0 & 1 & 0 \\
-2 & 2 & -3 & 2 & 0 & 0 \\
-2 & -1 & 3 & 1 & 1 & 0 \\
0 & 0 & 0 & -1 & -1 & 1
\end{array} \|
$$

and has the property that the first column of $B A$ is the same as the first column of the unit matrix. Moreover, the coefficients of $B$ are quite small, which is why there is little increase in the sizes of our numbers as yet.

We now seek to bring the second column of $B A$ into agreement with the second column of the unit matrix by means of elementary transformations. In order not to change the form of the first column, we must avoid the following transformations: (a) Multiplying the first row by a constant different from unity; (b) interchanging the first and $j$ th rows; (c) adding $\alpha$ times the first row to the $j$ th row for $\alpha \neq 0$.

However, the remaining transformations are quite adequate to effect the desired reduction. The second column of $B A$ is

$$
\begin{array}{r||}
-807 \\
1967 \\
981 \\
-891 \\
928 \\
79
\end{array}
$$

Confining attention to this column only, we see that the following sequence of transformations is called for. We add $\alpha$ times the $i$ th row to the $j$ th row for the following succession of $\alpha, i$, and $j$ :

\begin{tabular}{r|r|r|r|r|r|r|r|r|r|r|r|r|r|r|r|r|r|r|r|r|}
$\alpha$ & -2 & -1 & +1 & -1 & 11 & -1 & -1 & -1 & -1 & +1 & +1 & -1 & +1 & +1 & -1 & -1 & -1 & -1 & 4 & +1 \\
\hline$i$ & 3 & 5 & 4 & 4 & 6 & 6 & 3 & 5 & 6 & 4 & 3 & 5 & 4 & 2 & 2 & 2 & 2 & 6 & 4 & 2 \\
\hline$j$ & 2 & 3 & 5 & 1 & 4 & 1 & 6 & 3 & 5 & 6 & 4 & 3 & 5 & 4 & 1 & 3 & 5 & 2 & 6 & 4
\end{tabular}

Performing these transformations on $B$ gives $C$, namely,

$$
\begin{array}{||rrrrrr}
-2 & 6 & -5 & 0 & 7 & -1 \\
6 & -6 & 7 & 8 & 7 & -12 \\
3 & 5 & -4 & -6 & 2 & 1 \\
12 & -9 & 8 & -6 & -10 & -1 \\
-9 & 15 & -7 & -9 & -5 & 10 \\
23 & -13 & 1 & -65 & -80 & 56
\end{array} \|
$$


The first two columns of $C A$ are identical with those of the unit matrix. The third column is

$$
\begin{array}{r}
-491 \\
1399 \\
857 \\
2968 \\
-439 \\
5637
\end{array}
$$

In dealing with this, we must now curtail transformations on the first two rows. Nevertheless, we can reduce it to the desired form by adding $\alpha$ times the $i$ th row to the $j$ th row for the following succession of $\alpha$, and $j$ :

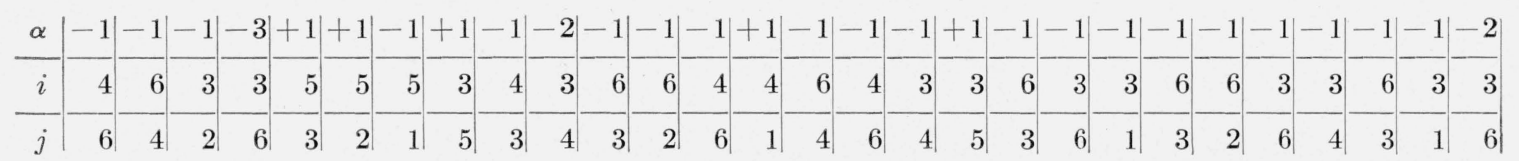

Performing these transformations on $C$ gives $D$, namely,

$$
\begin{array}{||rrrrrr}
-293 & 815 & -1464 & -5481 & -6270 & 5967 \\
65 & -187 & 350 & 1317 & 1509 & -1434 \\
283 & -796 & 1373 & 5057 & 5745 & -5493 \\
-68 & 158 & -351 & -1447 & -1721 & 1597 \\
-24 & 79 & -49 & -51 & 5 & 36 \\
-744 & 2095 & -3608 & -13278 & -15079 & 14421
\end{array} \|
$$

The first three columns of $D A$ are identical with those of the unit matrix. The fourth column is

$$
\begin{array}{r}
551061 \\
-133644 \\
-503483 \\
151308 \\
-2958 \\
1321462
\end{array}
$$

\begin{tabular}{|c|c|c|c|c|c|c|c|c|c|c|c|c|c|c|c|c|c|c|c|c|c|}
\hline$\alpha$ & -8 & -3 & +3 & +1 & -1 & -1 & -2 & +1 & -1 & -3 & +1 & -2 & +1 & +3 & -1 & -1 & +2 & +1 & +1 & +1 & +1 \\
\hline$i$ & 4 & 4 & 4 & 4 & 6 & 6 & 4 & 4 & 6 & 4 & 4 & 4 & 4 & 5 & 5 & 5 & 4 & 4 & 5 & 5 & 6 \\
\hline & 6 & 1 & 3 & 2 & 4 & 1 & 6 & 3 & 4 & 6 & 1 & 2 & 3 & 4 & $1 \mid$ & 2 & 5 & 1 & 4 & 2 & \\
\hline
\end{tabular}

In dealing with this, we must now curtail transformations on the first three rows. Nevertheless, we can start out to reduce it to the desired form by adding $\alpha$ times the $i$ th row to the $j$ th row for the following succession of $\alpha, i$, and $j$ :

\begin{tabular}{r|r|r|r|r|r|r|r|r|r|r|r|r|r|r|r|r|r|r|r|r|}
$\alpha$ & -1 & +2 & +1 & -3 & -1 & -1 & +1 & +1 & +1 & +1 & -1 & +1 & -1 & +1 & +1 & -3 & +1 & -2 & +1 & +2 \\
\hline$i$ & 6 & 5 & 4 & 6 & 6 & 6 & 6 & 5 & 5 & 4 & 4 & 4 & 6 & 5 & 4 & 6 & 6 & 6 & 6 & 5 \\
\hline$j$ & $-\frac{5}{3}$ & 6 & 5 & 4 & 3 & 1 & 2 & 6 & 3 & 5 & 1 & 2 & 4 & 6 & 5 & 4 & 1 & 2 & 3 & 6
\end{tabular}


We now have the fourth column reduced to

$$
\left|\begin{array}{r}
-1 \\
0 \\
-1 \\
0 \\
-2 \\
0
\end{array}\right|
$$

We cannot reduce the -2 in the fifth row to unity except by dividing by 2 , which would introduce fractions. We would prefer to delay the introduction of fractions until the last possible moment. Accordingly, we multiply the first and third rows by 2 , then subtract the fifth row from the first and third, then multiply the fifth row by -1 , and, finally, interchange the fourth and fifth rows.

We have now changed the first and third columns so that they are no longer the first and third columns of the unit matrix, but have a 2 where the unit matrix has a unity. Also our fourth column has the same property. This can readily be corrected by multiplying the appropriate rows by one-half, but we will postpone this step until we can no longer avoid fractions.

Performing the indicated transformations on $D$ gives the matrix

$$
\begin{array}{||rrrrrr}
-32262 & 147945 & -111285 & -133369 & -13466 & 99868 \\
-12391 & 60699 & -46328 & -54117 & -3115 & 39630 \\
3330 & -20337 & 16173 & 17547 & -1298 & -11972 \\
-36052 & 168729 & -127507 & -151577 & -13242 & 112718 \\
-14752 & 74554 & -57273 & -66137 & -2494 & 47933 \\
83708 & -393352 & 297521 & 353125 & 29905 & -262237
\end{array} \|
$$

This is not exactly the matrix $E$ as we defined it earlier, but is close enough so that we shall call it $E$. The first four columns of $E A$ are essentially the first four columns of the unit matrix, merely having 2 in place of unity in the first, third, and fourth columns. The fifth column of $E A$ is

$$
\begin{array}{r||}
22281663 \\
9455740 \\
-3473233 \\
25687577 \\
11787645 \\
-60010368
\end{array}
$$

\begin{tabular}{|c|c|c|c|c|c|c|c|c|c|c|c|c|c|c|c|c|c|c|c|}
\hline$\alpha$ & +5 & -2 & -2 & -1 & & 11 & -3 & -2 & + & & -1 & -181 & -43 & -37 & - & - & \begin{tabular}{l|l}
-5 & 7
\end{tabular} & \begin{tabular}{l|l}
3 & 3
\end{tabular} & -3 \\
\hline$i$ & 5 & 5 & 5 & 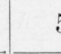 & & 6 & 6 & 6 & & ; & 6 & 5 & 5 & 5 & & 5 & 56 & $\begin{array}{ll}6 & 6\end{array}$ & 6 \\
\hline$j$ & 6 & 4 & 1 & 2 & 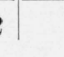 & 5 & 3 & 2 & & 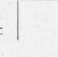 & 1 & 6 & 3 & 1 & & 2 & \begin{tabular}{l|l}
4 & 5
\end{tabular} & \begin{tabular}{l|l}
4 & 1
\end{tabular} & 2 \\
\hline$\alpha$ & +2 & $5 \mid$ & -2 & +2 & -2 & +1 & 2 & 1 & -1 & +1 & -1 & -1 & +1 & -1 & +4 & -1 & +2 & +1 & +1 \\
\hline$i$ & 6 & 5 & 5 & 5 & 5 & 5 & 6 & 6 & 6 & 5 & 5 & 5 & 6 & 6 & 5 & 5 & 6 & 6 & 6 \\
\hline$j$ & 3 & 6 & 2 & 1 & 3 & 4 & 5 & 2 & 3 & 6 & 4 & 2 & 5 & 1 & 6 & 1 & 5 & 3 & 4 \\
\hline
\end{tabular}

We now reduce this by adding $\alpha$ times the $i$ th row to the $j$ th row for the following succession of $\alpha, i$, and $j$ : 
We now multiply the second, third, and fourth rows by 3 , then add -1 times the sixth row to the second and +1 times the sixth row to the third and fourth. Finally, we interchange the fifth and sixth rows.

We could perform these transformations directly on $E$ to get a matrix that we will call $F$. However, it is computationally easier to proceed as follows. We perform the transformations on the unit matrix, getting the matrix.

$$
\begin{array}{|rrrrrr}
1 & 0 & 0 & 0 & 2953877 & 580221 \\
0 & 3 & 0 & 0 & 10836604 & 2128600 \\
0 & 0 & 3 & 0 & -34628689 & -6802003 \\
0 & 0 & 0 & 3 & -33984487 & -6675463 \\
0 & 0 & 0 & 0 & -9019777 & -1771726 \\
0 & 0 & 0 & 0 & -20003456 & -3929215
\end{array} \|
$$

Then, if we multiply $E$ on the left by the matrix above, we get the same matrix $F$ that we would get by performing the indicated transformations on the rows of $E$. This matrix $F$ follows.

\begin{tabular}{||rrrrrrr}
4993513702 & -8007596989 & 3450423435 & 9529844107 & 9984526301 & -10567128268 \\
18319229419 & -29376710487 & 12658240724 & 34961233901 & 36629283279 & -38766619778 \\
-58539637006 & 93874143339 & -40449780947 & -111719652341 & -117049953243 & 123879874958 \\
-57450612736 & 92127784365 & -39697285793 & -109641309887 & -114872450163 & 121575313514 \\
-15247889704 & 24451511094 & -10536003125 & -29099752301 & -30488142192 & 32267140121 \\
-33815746308 & 54226920056 & -23366040527 & -64535477403 & -67614555311 & 71559897507
\end{tabular}$\|$

The matrix $F A$ is

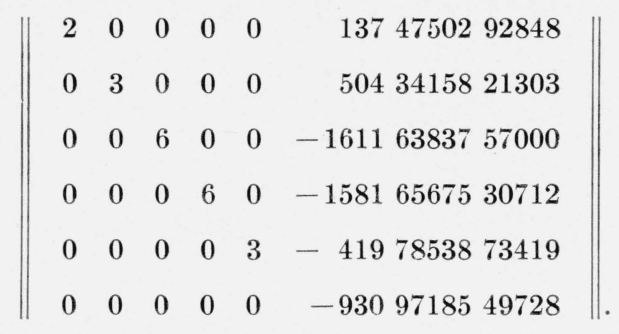

At this point, we can read off the determinant of $A$. Most of the transformations that we used in forming $F$ are such as to leave the determinant unchanged. Compiling those that do change the determinant, we find that the determinant of $F$ is 108 . So the determinant of $A$ is -55858311298368 .

One can readily write down the inverse of $F A$, namely, a matrix whose components are fractions with the common denominator 9309718549728 and the following numerators

\begin{tabular}{||cccccr||}
4654859274864 & 0 & 0 & 0 & 0 & 687375146424 \\
0 & 3103239516576 & 0 & 0 & 0 & 1681138607101 \\
0 & 0 & 1551619758288 & 0 & 0 & -2686063959500 \\
0 & 0 & 0 & 1551619758288 & 0 & -2636094588452 \\
0 & 0 & 0 & 0 & 3103239516576 & -1399284624473 \\
0 & 0 & 0 & 0 & 0 & -1
\end{tabular}


Finally, we compute $A^{-1}$ from the equation $A^{-1}=(F A)^{-1} F$. This gives the same matrix for $A)^{-1}$ that was computed in section 1 .

The computations outlined in section 2 required 23 hours of computing time by a professional computer. This time included the time needed to train the computer in the unfamiliar method.

\section{Remarks on Computational Details}

One advantage of the procedure outlined in section 2 is the ease in making numerical checks. Since all the operations are on rows, one can easily carry an extra check column which is the sum of all the columns with which one is dealing. However, this is not needed, for other checks are possible, as follows. The computation of $B$ is easily checked by computing the first column of $B A$ and seeing if it agrees with the first column of the unit matrix. Then one can check $C$ by computing the first two columns of $C A$ and seeing if they agree with the first two columns of the unit matrix; and so on.

Not only does this furnish a convenient check, but when a check is not forthcoming, one can often find the error by this method. For example, in computing $E$, two mistakes were made, and the resulting matrix was

$$
\begin{array}{||rrrrrr||}
-32262 & 147945 & -111285 & -132919 & -13466 & 99868 \\
-12391 & 60699 & -46328 & -54117 & -3115 & 38872 \\
3330 & -20337 & 16173 & 17997 & -1298 & -11972 \\
-36052 & 168729 & -127507 & -151127 & -13242 & 112718 \\
-14752 & 74554 & -57273 & -66587 & -2494 & 47933 \\
83708 & -393352 & 297521 & 352225 & 29905 & -262237
\end{array}
$$

which differs from $E$ in most elements of the fourth column (where the original error had snowballed) and in the second element of the sixth column. When we multiplied this matrix on the right by $A$, we got

$$
\begin{array}{r||}
70652 \\
-144778 \\
70650 \\
70650 \\
-70650 \\
-141300
\end{array}
$$

as the first column, instead of twice the first column of the unit matrix. So, except for the second row, our errors are all multiples of 70650, which is $(450) \times(157)$. As 157 is the fourth element in the first column of $A$, it seems clear that there are errors in the fourth column of what purports to be $E$, and that these errors are multiples of 450 . With this information, the mistake in computing in the fourth column was quickly discovered and eliminated. Now multiplication on the right by $A$ verified all but the second row, and a trivial amount of detective work on the second row of the product sufficed to locate the error in the second row and give its magnitude.

In setting up the computation for use on Iвм machinery, we notice that the majority of the steps consist of adding $\alpha$ times a row vector to another row vector, and that commonly $\alpha$ is a small integer. It is not difficult to wire a multiplier so that if we insert a deck with a card containing $\alpha$ followed by cards with the components of the two vectors interleaved, the multiplier will punch cards with the components of the resulting vector. At various stages in the procedure, some columns are computed for the product of two matrices, of which the first has as rows the row vectors that we are manipulating, and of which the second is always $\mathrm{A}$. 
Such a matrix multiplication can also be wired up for the multiplier. After the matrix multiplication, one then makes a list of $\alpha$ 's and rows to be operated on by inspection from a column. This is most conveniently done by hand, which permits the exercise of judgment at this point. However, one can proceed perfectly mechanically, as we did in the illustrative example. Indeed, it is not clear that one can really do much better by exercising judgment than we did with our purely mechanical procedure. Once the list of $\alpha$ 's and rows to be operated on is compiled, the respective operations can be quickly performed on Iвм machinery. With only a multiplier, one must keep each row as a deck, and the row decks have to be interleaved and separated repeatedly. If a card programmed calculator is available, one may put an entire row on a single card (unless the matrix is of really high order) and the operations are greatly speeded.

The matrix $A$ that we used was constructed from a table of random numbers in an effort to furnish an example that might be considered typical.

With an increase in the order of the matrix to be inverted, the method presented herein becomes even more of an improvement over the standard methods. The method was first devised in the summer of 1948 while working with Dr. N. G. Gunderson at Cornell University on a problem in number theory, in which we required the exact solution of 15 equations in 16 unknowns (one unknown was transposed to the right-hand side, and a solution obtained in terms of it). Fortunately, many of the coefficients were zeros. Even so, the usual methods of solution led us to hopelessly large numbers, whereas a solution was carried out by the method of this paper without encountering any integer of more than 12 digits.

Some of the procedural details of the present method were devised by Dr. Gunderson. The computations for the present paper were carried out by Lillian Forthal, Nancy Mann, and Gerald Kimble, under the direction of Marvin Howard.

Los Angeles, August 14, 1950. 